\title{
ARHGAP25 expression in colorectal cancer as a biomarker associated with favorable prognosis
}

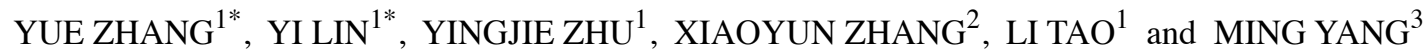 \\ Departments of ${ }^{1}$ Oncology and ${ }^{2}$ Pathology, Longhua Hospital; ${ }^{3}$ Phase I Clinical Research Laboratory of \\ Shanghai Longhua Hospital, Shanghai University of Traditional Chinese Medicine, Shanghai 200032, P.R. China
}

Received September 22, 2021; Accepted January 21, 2022

DOI: $10.3892 / \mathrm{mco} .2022 .2517$

\begin{abstract}
Although progress has been made in the early diagnosis of colorectal cancer (CRC) and in the systemic therapy of patients with CRC, the prognosis for advanced CRC remains poor. Our previous study demonstrated that ARHGAP25 overexpression significantly inhibits CRC cell growth, invasion and migration. However, it was not possible to evaluate and analyze the overall survival (OS) rate of patients with CRC. Thus, the discovery of relevant factors and their expression on the basis of existing research is necessary to predict the OS rate of patients with advanced CRC. Therefore, the aim of the present study was to define the value of Rho GTPase-activating protein 25 (ARHGAP25) expression in predicting the OS rate in patients with CRC. The clinical data of 153 patients with CRC who underwent colorectal resection were retrospectively analyzed. In order to explore the expression of ARHGAP25, immunohistochemical analysis of the tumor tissues of these patients, was performed. Univariate Cox regression analysis was used to assess the prognostic value of ARHGAP25 expression for OS. Multivariate analysis was used to evaluate the effect of ARHGAP25 expression in the presence of other variables. Confounding factors and interaction were assessed by a stratified analysis using ARHGAP25 expression and other variables associated with survival. The univariate analysis revealed that, ARHGAP25 expression was associated with an improved OS in patients with CRC $(\mathrm{P}<0.05)$. The multivariate analysis
\end{abstract}

Correspondence to: Dr Li Tao, Department of Oncology, Longhua Hospital, Shanghai University of Traditional Chinese Medicine, 725 Wanping South Road, Xuhui, Shanghai 200032, P.R. China E-mail: taoli20121228@163.com

Dr Ming Yang, Phase I Clinical Research Laboratory of Shanghai Longhua Hospital, Shanghai University of Traditional Chinese Medicine, 725 Wanping South Road, Xuhui, Shanghai 200032, P.R. China

E-mail: yangpluszhu@sina.com

*Contributed equally

Key words: Rho GTPase-activating protein 25, colorectal cancer, prognosis, overall survival, biomarker revealed that ARHGAP25 expression was still correlated with an improved OS after adjusting for sex, age, invasion degree, lymph node metastasis, distant metastasis, TNM stage, tumor location, histological type, histological grade, tumor deposits, and postoperative treatment $(\mathrm{P}<0.05)$. The stratified analysis demonstrated that the predictive value of ARHGAP25 for the OS of patients with CRC was stronger in males, elderly patients ( $>70$ years old), patients with T3 stage tumor, lymph node metastasis, TNM stage III, right hemicolon location and patients with a poorly differentiated tumor $(\mathrm{P}<0.05)$. Overall, our results demonstrated that ARHGAP25 may have an important potential value for improving the prognosis of patients with CRC.

\section{Introduction}

Colorectal cancer (CRC) is a serious threat to public health, as it ranks second in terms of mortality and third in terms of incidence. Over 1.8 million new colorectal cancer cases and 881,000 cancer-related deaths occurred in 2018, accounting for approximately 1 in 10 cancer cases and deaths (1). Although progress has been made in the early diagnosis of CRC and the systemic therapy, the prognosis for advanced CRC remains poor. Therefore, it is of utmost importance to identify new sensitive and specific biomarkers for the prognosis of patients with CRC.

The Rho GTPase-activating protein 25 (ARHGAP25) gene is located on the human chromosome $2 \mathrm{p} 13$ and encodes a protein of 639 amino acids with a potential Rho/Rac GAP domain (151-340 aa) (2). The ARHGAP25 protein is a member of the ARHGAP family and plays an important role in regulating $B$ cell chemotaxis and the germinal center reaction through the CXCL12/CXCR4 pathway (3). ARHGAP25 is present in leukocytes where it works as a negative regulator of the phagocytosis of neutrophils, and transendothelial migration of leukocytes $(4,5)$. Our previous work demonstrated that ARHGAP25 overexpression significantly inhibited CRC cell growth, suppressed cell migration and invasion, and reduced the expression of MMPs, EMT-associated factors and $\beta$-catenin. The expression of ARHGAP25 in colorectal cancer tissues was markedly lower than that in the normal adjacent tissue. In addition, the data of ARHGAP25 expression collected from both the GEO database and TCGA database indicated that ARHGAP25 mRNA expression was 
downregulated in patients with CRC when compared with the corresponding healthy controls (6). In the present study, the expression of ARHGAP25 in tumor tissues of patients with CRC was detected, and its association with the clinical characteristics and prognosis of patients was analyzed. Therefore, ARHGAP25 was used as a predictive target for prognosis, which is innovative and prospective.

\section{Materials and methods}

Patients. Patients who underwent colorectal tumor resection at the Longhua Hospital of Shanghai University of Traditional Chinese Medicine from February 24, 2009 to June 1, 2017, were included in this study. The main inclusion criteria were as follows: i) Patients who underwent resection of a large intestine tumor; ii) assessment of histologically proven colorectal cancer, staging and resectable specimens in accordance with the International Union against Cancer, 2017 (UICC) guidelines; and iii) patients who were well informed about the long-term follow-up. The main exclusion criteria were the following: i) Presence of another cancer; ii) presence of serious heart, liver or kidney complications; and iii) pregnant or lactating women, as well as children and individuals suffering from mental illness.

Ethical statement. The present study was approved by the Institutional Review Board of Longhua Hospital Shanghai University of Traditional Chinese Medicine (Approval no. 2018LCSY004). The study was performed in full agreement with the national ethical and regulatory guidelines, and with the 1964 Declaration of Helsinki and its later amendments or comparable ethical standards. Informed consent was obtained from all participants included in the present study. All patients signed a written informed consent to participate in this study.

Tissue sample collection. A sample of fresh tissue (including surgical and bioptic tissue) was selected; the collected CRC tissue sample included the malignant tumor tissue and the adjacent tissue surgically removed or obtained from the biopsy. After the surgery, the tissue samples were collected under the guidance of clinical tumor pathologists to avoid misdiagnosis, with the following citeria: a) Sampling sites included colorectal cancer tissue and adjacent tissue (the latter 1-3 cm away from the cancerous tissue); b) clear clinical diagnosis with the removal of the specimens without radiotherapy or chemotherapy performed under the guidance of pathologists; c) the survival of the cancerous tissue was ensured; d) the surgically removed tissue samples were quickly submerged into liquid nitrogen and then stored in a liquid nitrogen tank or at $-80^{\circ} \mathrm{C}$ within $30 \mathrm{~min}$ from the collection of the surgical specimen; e) images of the samples were obtained using a digital camera; then, they were cut into small pieces but not too small (size $0.5 \times 0.5 \mathrm{~cm}$, thickness $<0.5 \mathrm{~cm}$ ), placed into the frozen storage tube, and into the specimen box. The label was attached on the lid to record the specimen number, collection time and specimen type. A total of 3-5 cryopreservation tubes containing the specimens were stored for each patient, and each tissue weighed no less than $2 \mathrm{~g}$.
Expression of ARHGAP25. Immunohistochemistry (IHC) was performed to assess ARHGAP25 expression. Specific steps were carried out according to the instructions of the immunohistochemistry kit. In brief, antigen repair was performed using $0.01 \mathrm{M}$ sodium citrate buffer at high pressure for $15 \mathrm{~min}$, followed by cooling for $5 \mathrm{~min}$ and washing with PBS buffer 3 times for $3 \mathrm{~min}$. Subsequently, 10\% goat serum (diluted 20 times with PBS) (cat. no. SL038; Beijing Solarbio Science \& Technology Co., Ltd.) was directly added to the 4-mm thick tissue sections and incubated at $37^{\circ} \mathrm{C}$ for $10 \mathrm{~min}$. They were then incubated overnight with the primary antibody against ARHGAP25 (diluted 1:200; rabbit IgG1; product code ab192020; Abcam) at $4^{\circ} \mathrm{C}$. Next, they were incubated with the secondary antibody (diluted 1:1,000; product no. D-3004-100; Shanghai Long Island Biotech Co., Ltd.) at $25^{\circ} \mathrm{C}$ for $30 \mathrm{~min}$. Finally, the slides were incubated with DAB staining solution at room temperature for $15 \mathrm{~min}$ (product no. FL-6001-03; Shanghai Long Island Biotech Co., Ltd.) until the required staining levels were reached and counterstained with hematoxylin for $3 \mathrm{~min}$ (product code BA4097; BASO), followed by differentiation with $1 \%$ alcohol hydrochloric acid and light microscopic observation to control the degree of staining. The sections were then rinsed with running water for $10 \mathrm{~min}$ and placed in an oven at $65^{\circ} \mathrm{C}$ to remove all moisture. The slides were observed under an Olympus inverted light microscope at a magnification of $\mathrm{x} 200$. The expression of ARHGAP25 was mainly observed in the cytoplasm of the tissue cells as brownish yellow particles. The expression of ARHGAP25 was subjected to $\log _{2}$ transformation before the statistical analysis. The median method was used to classify colorectal cancer as high and low expression of ARHGAP25, and the median value was 3.07. All samples were divided into high and low ARHGAP25 groups, with the median ARHGAP25 value as the cutoff point: The ARHGAP25-high expression group was the one with an expression higher than the median value, the ARHGAPDH25-low expression group was the one with an expression lower than the median value.

Semi-quantification of IHC. A simple method of automated digital IHC image analysis algorithm was used for an unbiased, quantitative assessment of the intensity of the antibody staining in the tissue sections. The IHC Profiler, an open source plugin for the quantitative evaluation and automated scoring of IHC images of human tissue samples was used to calculate the positive area of CRC tissue and adjacent tissue. The IHC Profiler, which is compatible with the ImageJ software version 1.53 (National Institutes of Health), performs IHC analysis using color deconvolution and computerized pixel profiling leading to the assignment of an automated score to the respective image. This method used in the present study has been thoroughly validated using high volume IHC digital datasets representing multiple protein markers expressed in the cytoplasm $(7,8)$.

Main outcome. The primary outcome was the overall survival (OS), defined as the the date of surgical resection of the colorectal tumor until death from any cause. The follow-up was based on appointments, scheduled questionnaires, and telephone surveys, and was carried out until the end of the study on May 31, 2020, which served as the establishment of an outcome and endpoints. 
Variables. The medical records and electronic records of the patients were analyzed, and the following parameters were collected: Demographic factors (age and sex), tumor-related factors (degree of invasion, lymph node metastasis, distant metastasis, TNM stage, nerve invasion, vascular invasion, resection edge, tumor deposition, tumor location, histological type and histological grade), expression of the tumor-associated protein ARHGAP25 and postoperative treatment radiotherapy, chemotherapy, targeted therapy and traditional Chinese medicine (TCM).

Statistical analysis. A total of 153 patients with colorectal cancer were included in this study, and among these, 124 had complete data and no missing values. The median follow-up time was 54.9 months [interquartile range (IQR): 27.30, 60.33]. Among the 153 eligible patients, $42(27.5 \%)$ succumbed to CRC, 77 $(50.3 \%)$ survived, and the remaining 34 were lost to follow-up.

The scatter diagram was plotted using the GraphPad Prism 8.0.2 software (GraphPad Software, Inc.), and the statistical significance was analyzed by paired t-test. The OS curve was plotted using the Kaplan Meier method, and the statistical significance was calculated by log-rank test and Gehan Breslow Wilcoxon test. The aforementioned data were analyzed by GraphPad Prism 8.0.2 software.

Cox proportional hazards regression analysis was used for univariate analysis, multivariate analysis and hierarchical analysis. Five models based on variable adjustment were constructed in multivariate analysis: M0, no variable; M1, age, sex; M2, degree of invasion, lymph node metastasis, distant metastasis, TNM stage; M3, tumor location, histological type, histological grade, tumor deposition; M4, chemotherapy, TCM treatment; M5, age, sex, degree of invasion, lymph node metastasis, distant metastasis, TNM stage, tumor location, histological type, histological grade, tumor deposition, chemotherapy, TCM treatment. The risk ratio (HR) with $95 \%$ confidence interval (CI) and two-sided P-values were calculated. Variables containing $>20 \%$ missing values and zero variance were deleted. The present study used three methods to overcome the missing values $(<20 \%)$ : Complete case analysis, multiple interpolation and missing index method (9-11). Values of $\mathrm{P}<0.05$ were considered to indicate a statistically significant difference. The aforementioned data were analyzed by the software $\mathrm{R}$ programming language, $\mathrm{R}$ version 3.5.3 (https://www.r-project.org/).

\section{Results}

ARHGAP25 expression in CRC and tumor adjacent tissues. The results of IHC on 26 pairs of CRC tissues and corresponding adjacent tissues revealed that the expression of ARHGAP25 in the adjacent tissues was higher than that in the CRC tissues $(\mathrm{P}=0.0016)$. The statistical difference was analyzed by paired t-test (Fig. 1A). Moreover, the sample size was increased by the addition of $127 \mathrm{CRC}$ tissue samples, for a total of $153 \mathrm{CRC}$ tissues that were subjected to IHC: a) Adjacent tissues (ARHGAP25), the ARHGAP25-positive area was 2,785; b) CRC tissue (low ARHGAP25), the ARHGAP25-positive area was 520; c) CRC tissue (high ARHGAP25), the ARHGAP25-positive area was 1,001; d) adjacent tissues (ARHGAP25), ARHGAP25-positive area was 3,448; e) CRC tissue (low ARHGAP25), the
ARHGAP25-positive area was 280; and f) CRC tissue (high ARHGAP25), the ARHGAP25-positive area was 1,071. The magnification was x200 (Fig. 1B).

Patient characteristics. A total of 153 patients suffering from colorectal cancer were enrolled in the present study, and among them, 124 had complete data with statistical analysis and no missing values. The details of the baseline characteristics are listed in Table I.

Correlation between ARHGAP25 expression and OS. The total 153 patients with CRC were divided into two subgroups according to the low and high ARHGAP25 expression, which accounted for $49.67 \%$ (76/153) and $50.33 \%$ (77/153), respectively. The OS of patients with CRC with high expression of ARHGAP25 was significantly higher than that of the patients with low expression of ARHGAP25. The median survival of the patients with high expression of ARHGAP25 was 60.13 months, and the median survival with those with low expression of ARHGAP25 was 53.87 months $(\mathrm{P}<0.05)$ (Fig. 2). A high expression of ARHGAP25 indicated a favorable prognosis of patients with CRC. The OS curve of ARHGAP25 revealed that the survival time of patients with high expression of ARHGAP25 was significantly longer than that of patients with low expression of ARHGAP25 (Fig. 2).

Univariate analysis. Univariate Cox analysis was used to evaluate the prognostic value of ARHGAP25 expression and other factors affecting the survival of patients with CRC using complete data. The results revealed that the expression of ARHGAP25 was associated with the improvement of OS in patients with $\mathrm{CRC}(\mathrm{P}=0.011 ; \mathrm{HR}, 0.213 ; 95 \% \mathrm{CI}, 0.065-0.701)$. In addition, age $(\mathrm{P}=0.012 ; \mathrm{HR}, 1.051 ; 95 \% \mathrm{CI}, 1.011-1.093)$, lymph node metastasis ( $\mathrm{P}<0.001 ; \mathrm{HR}, 5.312 ; 95 \% \mathrm{CI}$, 2.191-12.879), distant metastasis $(\mathrm{P}=0.007$; HR, 5.529; $95 \%$ CI, 1.584-19.295), TNM stage $(\mathrm{P}=0.0205$; HR, 4.356; 95\% CI, 1.254-15.13), signet ring cell carcinoma $(\mathrm{P}=0.001 ; \mathrm{HR}, 13.625$; 95\% CI, 3.025-61.366), and tumor deposition ( $\mathrm{P}=0.011$; HR, $3.566 ; 95 \%$ CI, 1.333-9.538) were associated with adverse OS in patients with CRC (Table II).

Multivariate analysis. Multivariate analysis was performed to determine whether the expression of ARHGAP25 was still associated with OS after adjusting for other variables. The complete case analysis revealed the following results: M0 (adjusted $\mathrm{P}=0.011$; adjusted HR, 0.213; 95\% CI, 0.065-0.701), M1 (adjusted $\mathrm{P}=0.017$; adjusted HR, 0.238; $95 \%$ CI, 0.073-0.777), M2 (adjusted $\mathrm{P}=0.024$; adjusted HR, 0.168; 95\% CI, 0.036-0.789), M3 (adjusted $\mathrm{P}=0.034$; adjusted HR, 0.220 ; 95\% CI, 0.055-0.889), M4 (adjusted $\mathrm{P}=0.012$; adjusted HR, 0.221; 95\% CI, 0.068-0.721) and M5 (adjusted P=0.003; adjusted HR, 0.096; 95\% CI, 0.021-0.449) (Fig. 3).

Multiple imputation analysis revealed the following results: M0 (adjusted $\mathrm{P}=0.009$; adjusted HR, 0.280; 95\% CI, 0.108-0.724); M1 (adjusted $\mathrm{P}=0.008$; adjusted HR, 0.280; $95 \%$ CI, 0.110-0.716); M2 (adjusted $\mathrm{P}=0.012$; adjusted HR, 0.244; 95\% CI, 0.081-0.735); M3 (adjusted $\mathrm{P}=0.007$; adjusted HR, 0.246; 95\% CI, 0.089-0.680); M4 (adjusted $\mathrm{P}=0.009$; adjusted HR, 0.283; 95\% CI, 0.110-0.728); M5 (adjusted $\mathrm{P}<0.001$; adjusted HR, 0.106; 95\% CI, 0.030-0.369) (Fig. 3). 


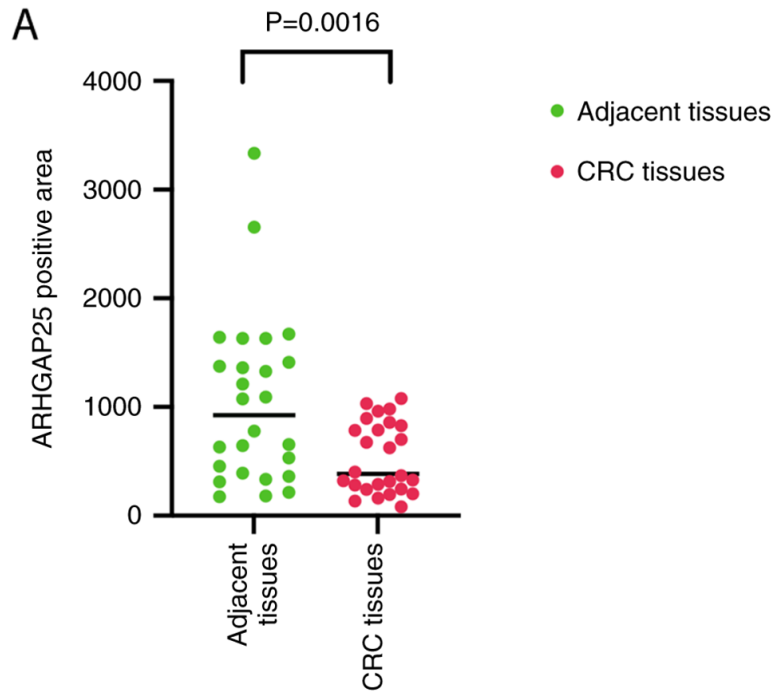

B a

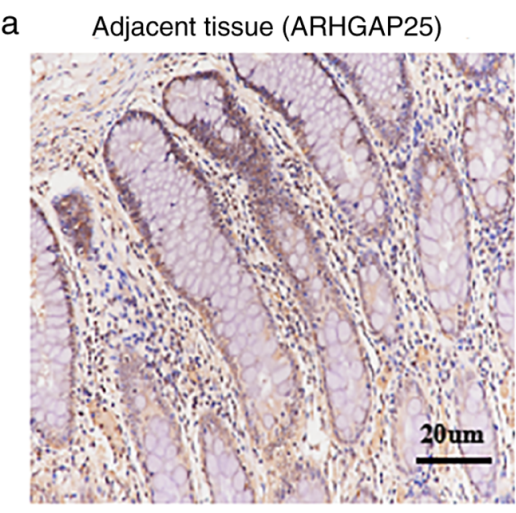

d

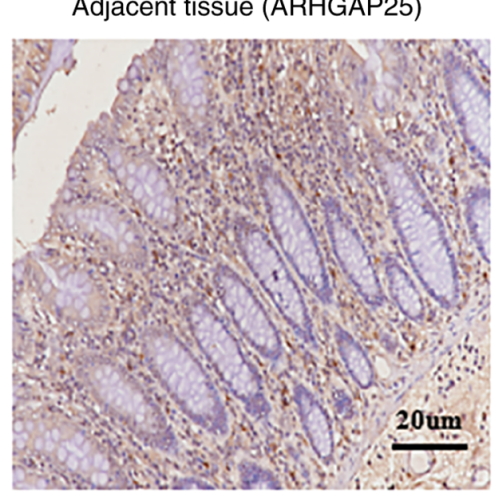

b

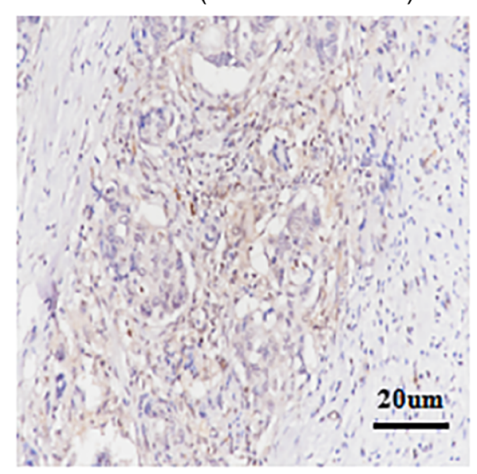

e

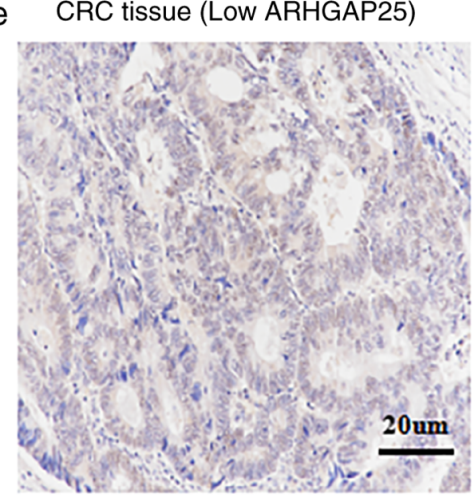

C CRC tissue (High ARHGAP25)

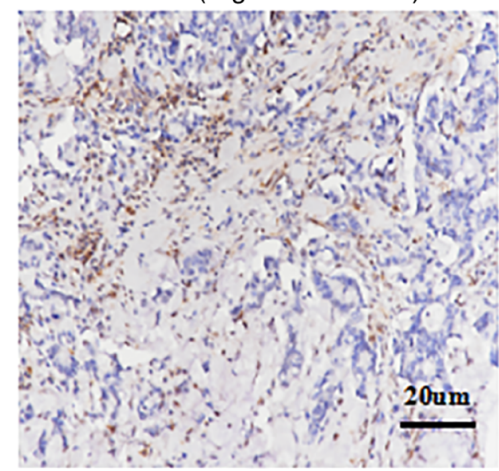

f CRC tissue (High ARHGAP25)

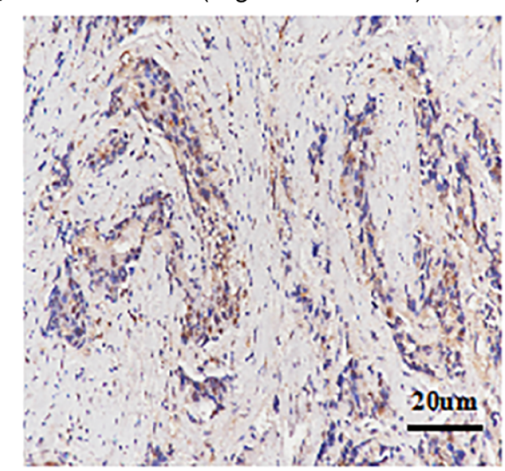

Figure 1. (A) IHC on 26 pairs of CRC tissues and corresponding adjacent tissues showing that the expression of ARHGAP25 in the adjacent tissues was higher than that in the CRC tissues ( $\mathrm{P}=0.0016)$. The statistical difference was evaluated by paired t-test. (B) The expression of ARHGAP25 in CRC and tumor adjacent tissues was detected with IHC, dividing the patients into low and high ARHGAP25 expression. (a) adjacent tissues (ARHGAP25), the ARHGAP25-positive area was 2,785; (b) CRC tissue (low ARHGAP25), the ARHGAP25-positive area was 520; (c) CRC tissue (high ARHGAP25), the ARHGAP25-positive area was 1,001; (d) adjacent tissues (ARHGAP25), the ARHGAP25-positive area was 3,448; (e) CRC tissue (low ARHGAP25), the ARHGAP25-positive area was 280; and (f) CRC tissue (high ARHGAP25), the ARHGAP25-positive area was 1,071. Original magnification, x200. IHC, immunohistochemistry; ARHGAP25, Rho GTPase-activating protein 25; CRC, colorectal cancer; IQR, interquartile range.

The analysis of missing index cases resulted in the following results: $\mathrm{M} 0$ (adjusted $\mathrm{P}=0.008$; adjusted $\mathrm{HR}, 0.280 ; 95 \% \mathrm{CI}$, 0.109-0.722); M1 (adjusted $\mathrm{P}=0.008$; adjusted HR, 0.283; 95\% CI, 0.111-0.722); M2 (adjusted $\mathrm{P}=0.012$; adjusted HR, 0.244; 95\% CI, 0.081-0.736); $\mathrm{M} 3$ (adjusted $\mathrm{P}=0.010$; adjusted HR, 0.267; 95\% CI, 0.098-0.727); M4 (adjusted $\mathrm{P}=0.746$; adjusted HR, 0.280; 95\% CI, 0.108-0.728); M5 (adjusted $\mathrm{P}=0.005$; adjusted HR, 0.220; 95\% CI, 0.077-0.632) (Fig. 3).
The addition of different grouping variables still revealed that ARHGAP25 expression was associated to the improvement of OS, indicating that ARHGAP25 is a protective factor to prevent poor prognosis in patients with CRC.

Stratified analysis. The analysis with complete cases revealed that the predictive value of ARHGAP25 for OS in patients with CRC was stronger in males, elderly patients ( $>70$ years old), T3 
Table I. Baseline characteristics of patients with colorectal cancer.

\begin{tabular}{lcc}
\hline Variables & Overall & Missing \\
\hline Age, median (IQR) & $69.00(61.00,79.00)$ & 0 \\
ARHGAP25, median (IQR) & $3.07(2.86,3.21)$ & 0 \\
OS, median (IQR) & $45.27(27.30,60.33)$ & 0 \\
Sex (\%) & & 0 \\
Male & $93(60.78)$ & \\
Female & $60(39.22)$ &
\end{tabular}

Invasion degree (\%)

$\mathrm{T} 1$

$\mathrm{T} 2$

$6(3.92)$

$36(23.53)$

$65(42.49)$

$45(29.41)$

T4

Lymph node metastasis (\%)

No

87 (56.86)

$66(43.14)$

Tumor deposits (\%)

No

Yes

Distant metastasis (\%)

No

Yes

TNM stage (\%)

I

II

III

IV

Tumor location (\%)

Right hemicolon

Left hemicolon

Rectum

$1(0.65)$

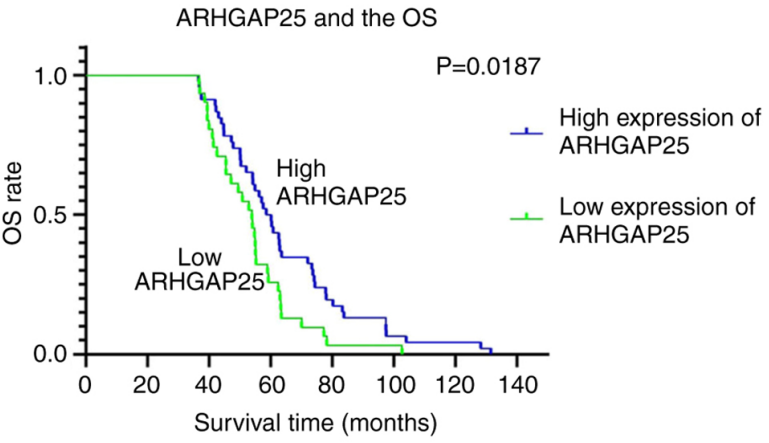

Figure 2. Association between ARHGAP25 expression and the OS. The OS curves were displayed with Kaplan-Meier method based on the ARHGAP25 expression. The statistical difference between the OS curves was analyzed with the log-rank test. ARHGAP25, Rho GTPase-activating protein 25; OS, overall survival.

0

0

$138(90.20)$

$15(9.80)$

$142(92.81)$

$10(6.54)$

$1(0.65)$

$39(25.49)$

$42(27.45)$

$61(39.87)$

$11(7.19)$

$40(26.14)$

$46(30.07)$

67 (43.79)

Histological subtype (\%)

Adenocarcinoma

$115(75.16)$

$30(19.61)$

3 (1.96)

5 (3.27)

Signet-ring cell carcinoma

Other

Histological grade (\%)

Well differentiated

Moderately differentiated

Poorly differentiated

Undifferentiated

Chemotherapy (\%)

No

Yes

TCM treatment (\%)

$\begin{array}{ll}\text { No } & 51(33.33) \\ \text { Yes } & 75(49.02)\end{array}$

$1(0.65)$

$130(84.97)$

$17(11.11)$

$1(0.65)$

$27(17.65)$

$27(17.65)$

T1, tumor invades the submucosa (through the muscularis mucosa but not into the muscularis propria); $\mathrm{T} 2$, tumor invades the muscularis propria; T3, tumor invades through the muscularis propria into pericolorectal tissues; T4, tumor invades through the visceral peritoneum or invades or adheres to adjacent organs or structures. stage, lymph node metastasis, TNM stage III, right hemicolon tumor location and poor differentiation $(\mathrm{P}<0.05)$. In addition, ARHGAP25 expression had a significant effect on patients with CRC without distant metastasis, without tumor deposits and without having received TCM treatment $(\mathrm{P}<0.05)$.

The analysis with missing-indicator cases produced results similar to those of the analysis with complete cases. In fact, it was revealed that ARHGAP25 expression was relevant to OS in elderly patients with CRC (>70 years old), males, T3-4 stage, lymph node metastasis, TNM stage III and poor differentiation $(\mathrm{P}<0.05)$. Additionally, ARHGAP25 expression was a significant predictor of OS in patients without distant metastasis, without tumor deposits and without having received TCM treatment $(\mathrm{P}<0.05)$.

The analysis with multiple imputation cases also revealed that ARHGAP25 expression was associated to OS in patients with $C R C>70$ years and male $(\mathrm{P}<0.05)$. Moreover, ARHGAP25 expression had a significant effect on patients with CRC with T3-4 stage, lymph node metastasis (adjusted $\mathrm{P}=0.007)$, TNM stage III and poorly differentiated adenocarcinoma $(\mathrm{P}<0.05)$. Additionally, ARHGAP25 expression was a significant predictor of OS in patients without distant metastasis, without tumor deposits, who received chemotherapy and without having received TCM treatment $(\mathrm{P}<0.05)$ (Table III).

\section{Discussion}

CRC is a huge global burden in terms of complications, mortality, side effects after the treatment, use of health care services, and medical costs (12). Therefore, the use of clinical samples to identify more new prognostic and predictive biomarkers is of great significance.

Rac/Rho-like GTPases are important regulators of several different cell functions, including cell polarity control, membrane transport, transcription regulation, survival, adhesion, and proliferation. GTPase-activating proteins (GAP) are negative regulators of Rac/Rho-like GTPase because they convert thes active GTP binding state to the inactive GDP binding state $(13,14)$. According to the homologous catalytic domain, approximately 70 proteins act as Rac/Rho GAP in different human tissues $(15,16)$. ARHGAP25 protein is a Rac-specific GAP mainly expressed in hematopoietic cells. 
Table II. Univariate analysis of the prognostic value of ARHGAP25 expression and other prognostic factors for predicting overall survival.

\begin{tabular}{|c|c|c|c|c|}
\hline Variables & No. of patients & HR & $95 \% \mathrm{CI}$ & P-value \\
\hline ARHGAP25 & 124 & 0.213 & $0.065-0.701$ & 0.011 \\
\hline Age & 124 & 1.051 & $1.011-1.093$ & 0.012 \\
\hline \multicolumn{5}{|l|}{ Sex } \\
\hline Male & 75 & NA & NA & NA \\
\hline Female & 49 & 1.542 & $0.702-3.387$ & 0.281 \\
\hline \multicolumn{5}{|l|}{ Invasion degree } \\
\hline $\mathrm{T} 1$ & 6 & NA & NA & NA \\
\hline $\mathrm{T} 2$ & 30 & 0.966 & $0-\operatorname{Inf}$ & 0.999 \\
\hline $\mathrm{T} 3$ & 58 & $4.63 \mathrm{E}+08$ & $0-\operatorname{Inf}$ & 0.999 \\
\hline $\mathrm{T} 4$ & 30 & $2.22 \mathrm{E}+08$ & $0-\operatorname{Inf}$ & 0.999 \\
\hline \multicolumn{5}{|l|}{ Lymph node metastasis } \\
\hline No & 76 & NA & NA & NA \\
\hline Yes & 48 & 5.312 & $2.191-12.879$ & $<0.001$ \\
\hline \multicolumn{5}{|l|}{ Tumor deposits } \\
\hline No & 115 & NA & NA & NA \\
\hline Yes & 9 & 3.566 & $1.333-9.538$ & 0.011 \\
\hline \multicolumn{5}{|l|}{ Distant metastasis } \\
\hline No & 117 & NA & NA & NA \\
\hline Yes & 7 & 5.529 & $1.584-19.295$ & 0.007 \\
\hline \multicolumn{5}{|l|}{ TNM stage } \\
\hline Non-IV & 116 & NA & NA & NA \\
\hline IV & 8 & 4.356 & $1.254-15.13$ & 0.0205 \\
\hline \multicolumn{5}{|l|}{ Tumor location } \\
\hline Right hemicolon & 33 & NA & NA & NA \\
\hline Left hemicolon & 41 & 0.387 & $0.14-1.071$ & 0.068 \\
\hline Rectum & 50 & 0.459 & $0.186-1.133$ & 0.091 \\
\hline \multicolumn{5}{|l|}{ Histological type } \\
\hline Adenocarcinoma & 92 & NA & NA & NA \\
\hline Mucinous adenocarcinoma & 27 & 1.865 & $0.751-4.63$ & 0.179 \\
\hline Signet ring cell carcinoma & 2 & 13.625 & $3.025-61.366$ & 0.001 \\
\hline Other & 3 & 19.727 & $3.907-99.592$ & $<0.001$ \\
\hline \multicolumn{5}{|l|}{ Histological grade } \\
\hline Well & 1 & NA & NA & NA \\
\hline Moderate & 107 & 2443062 & $0-\operatorname{Inf}$ & 0.997 \\
\hline Poor & 15 & 8535588 & $0-\operatorname{Inf}$ & 0.997 \\
\hline Undifferentiated & 1 & $1.9 \mathrm{E}+08$ & $0-\operatorname{Inf}$ & 0.997 \\
\hline \multicolumn{5}{|l|}{ Chemotherapy } \\
\hline No & 42 & NA & NA & NA \\
\hline Yes & 82 & 1.105 & $0.474-2.577$ & 0.817 \\
\hline \multicolumn{5}{|l|}{ TCM treatment } \\
\hline No & 51 & NA & NA & NA \\
\hline Yes & 73 & 0.661 & $0.299-1.463$ & 0.307 \\
\hline
\end{tabular}

ARHGAP25, Rho GTPase-activating protein 25; NA, not available; Inf, infinity.

The RhoE/ROCK/ARHGAP25 signaling pathway has been revealed to control the invasion potential of alveolar rhabdomyosarcoma cells (17). In addition, ARHGAP25 was revealed to be downregulated in lung cancer and inhibit its growth, migration and invasion through its effect on the Wnt/ $\beta$-catenin signaling pathway (18). 


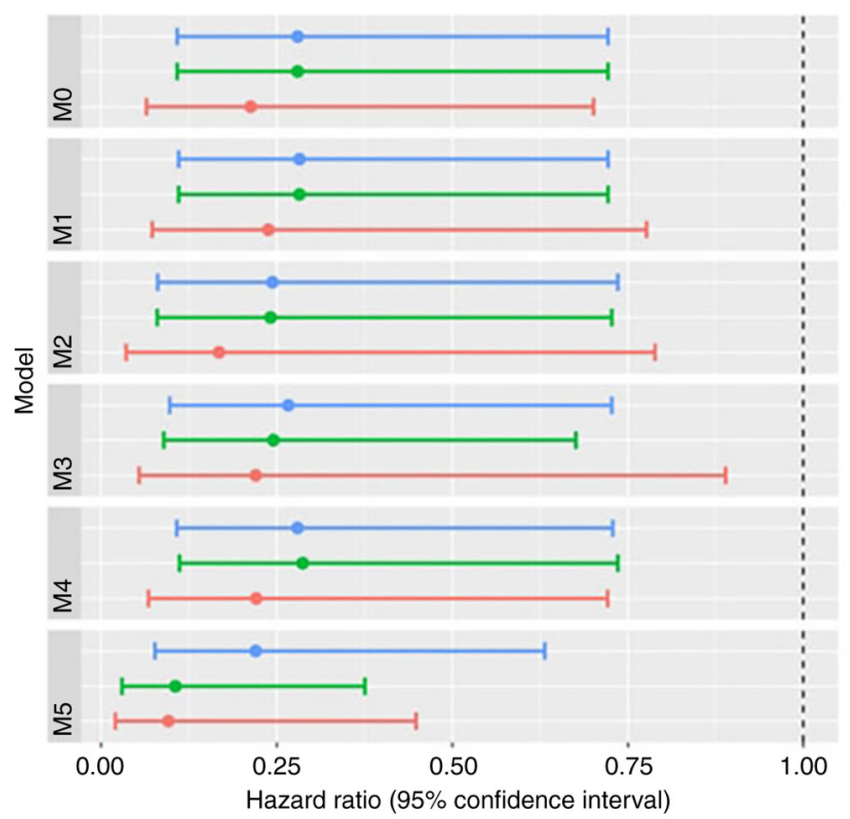

\begin{tabular}{lccc} 
& $\mathrm{n}$ & Events & $\mathrm{HR}\{95 \% \mathrm{Cl}\}$ \\
\cline { 2 - 4 } & 153 & 153 & $0.280(0.109-0.722)$ \\
& 153 & 153 & $0.280(0.108-0.724)$ \\
& 153 & 124 & $0.213(0.065-0.701)$ \\
& 153 & 153 & $0.283(0.111-0.722)$ \\
+ DataSet & 153 & 153 & $0.280(0.110-0.716)$ \\
+ Imputation & 153 & 124 & $0.238(0.073-0.777)$ \\
+ Missing-indicator & 153 & 153 & $0.244(0.081-0.736)$ \\
& 153 & 153 & $0.246(0.089-0.680)$ \\
& 153 & 153 & $0.220(0.055-0.889)$ \\
& 153 & 153 & $0.244(0.081-0.735)$ \\
& 153 & 153 & $0.280(0.108-0.728)$ \\
& 153 & 153 & $0.283(0.110-0.728)$ \\
& 153 & 124 & $0.221(0.068-0.721)$ \\
& & & \\
& 153 & 153 & $0.220(0.077-0.632)$ \\
& 153 & 153 & $0.106(0.030-0.369)$ \\
& 153 & 124 & $0.096(0.021-0.449)$
\end{tabular}

Figure 3. Forest plot of the multivariate analysis data of the prognostic value of ARHGAP25 expression for predicting overall survival in patients with colorectal cancer. M0, no variables; M1, age and sex; M2, invasion degree, lymph node metastasis, distant metastasis and TNM stage; M3, tumor location, histological type, histological grade and tumor deposits; M4, chemotherapy and TCM treatment; M5, age, sex, invasion degree, lymph node metastasis, distant metastasis, TNM stage, tumor location, histological type, histological grade, tumor deposits, chemotherapy and TCM treatment. ARHGAP25, Rho GTPase-activating protein 25; TCM, traditional Chinese medicine.

Our previous study (6) showed that the downregulation of ARHGAP25 in CRC was associated to the progression of CRC, and the upregulation of ARHGAP25 reduced CRC metastasis in vitro and in vivo. In addition, ARHGAP25 inhibited the migration and invasion of CRC cells by inhibiting the Wnt/ $\beta$-catenin pathway, reduced the expression of MMPs and inhibited EMT. Moreover, ARHGAP25 was downregulated in the colon of patients with CRC compared with normal adjacent tissues (6). Despite this evidence, more studies are required to further determine the prognostic value of ARHGAP25 expression. Therefore, the present study aimed to evaluate the importance of ARHGAP25 expression in predicting the outcome of patients with CRC.

The present study showed that ARHGAP25 expression was significantly associated with a favorable prognostic value when analyzed as a continuous variable. Our results indicated that older age, lymph node metastasis, distant metastasis, signet ring cell carcinoma, and tumor deposition were particularly associated with poor OS, which was also consistent with previous studies (19-21).

Multivariate analysis showed that ARHGAP25 was associated with the improvement of the prognosis of patients with $\mathrm{CRC}$ after adjusting data, suggesting that ARHGAP25 is an independent prognostic factor for patients with CRC.

Further stratified analysis of the complete case analysis revealed that ARHGAP25 expression significantly predicted OS, especially in the elderly and male patients. A previous study revealed that elderly patients ( $>75$ years) had worse survival outcomes than younger patients, because younger patients can receive surgery, radiotherapy, and chemotherapy, while older patients usually receive palliative care (22). A population-based study conducted in Germany confirmed the survival advantage of women compared with men with CRC (23). The present study similar to other studies also showed that ARHGAP25 expression had a significant predictive effect on OS in patients with T3 stage, lymph node metastasis, TNM stage III, right-sided colon cancer, and poorly differentiated patients. T3-4 staging is a risk factor for the prognosis of CRC (24-26), and lymph node metastasis is an independent prognostic factor for patients with CRC (27-29). An analysis of patients based on disease stages showed that patients with early tumors have a higher survival rate than patients with a tumor in an advanced stage (30). Recently, evidence revealed differences in molecular, pathological and clinical features between right-sided colon cancer and left-sided colon cancer (31). In the wild-type RAS population of CRYSTAL and FIRE-3, the prognosis of patients with left-sided colon tumors was significantly better than that of patients with right-sided colon tumors (30). In addition, patients with poor differentiation have significantly worse OS $(32,33)$. Our study revealed that ARHGAP25 expression was an important predictor of OS, especially in patients without tumor deposits. However, previous studies have revealed that the presence of tumor deposits is an independent risk factor in the prognosis of patients with CRC $(34,35)$. This bias in the data may be caused by the use of a small number of cases with tumor deposits in our study. In addition, our analysis showed that ARHGAP25 expression had a prognostic significance in the patients who did not receive TCM treatment. Our previous study revealed that TCM treatment is a protective factor for OS (36).

Similarly, the missing index method and multiple imputation data analysis revealed that ARHGAP25 expression significantly predicted OS, especially in the elderly, men, T3 


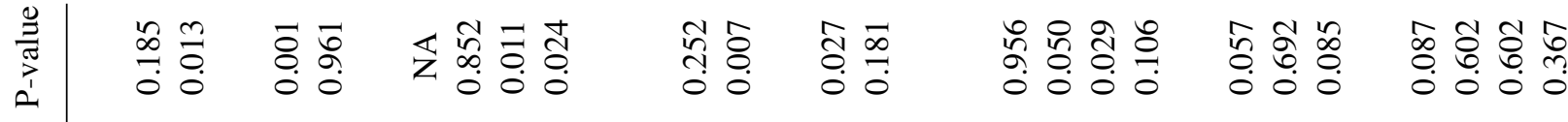

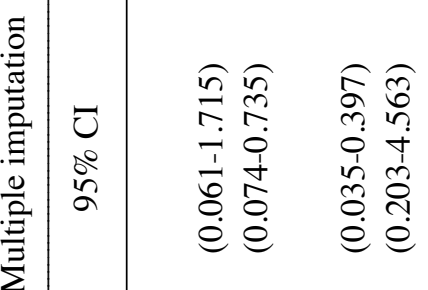

के

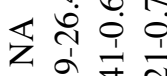

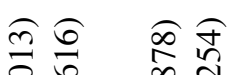

ลิจิธิด

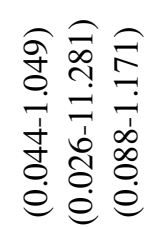

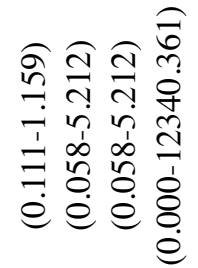

तै त़

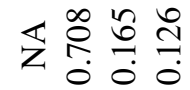

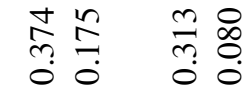

ஓํํำ

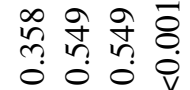

퓽

$\infty$

ต

ตถู่า

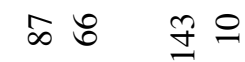

ले ริธี

안ํํำ

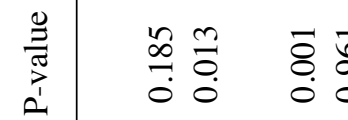

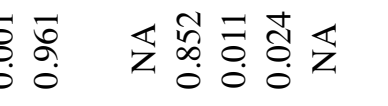

กิ

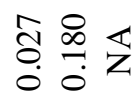

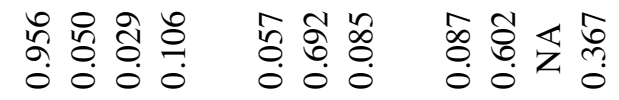

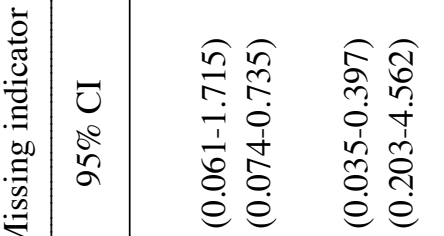

के

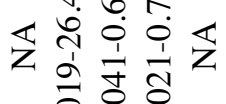

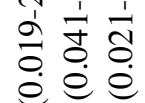

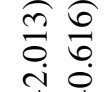

î

号

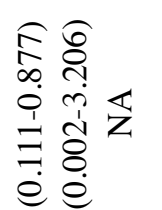

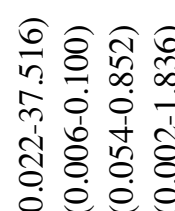

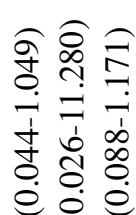

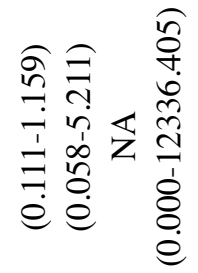

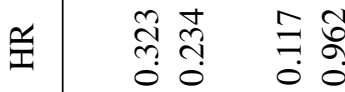

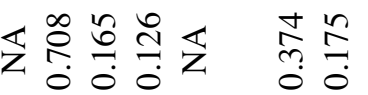

ติ

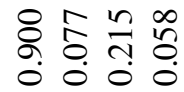

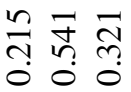

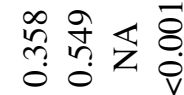

స్ㅠㅇ

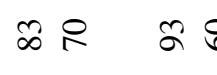

ช ๒

s。

은으-

ले ๆ

우운

$\cong 8 m n$

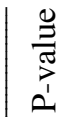

\begin{tabular}{ll}
8 & 0 \\
\hline & 0 \\
0 & 0
\end{tabular}

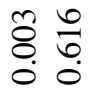

不

के

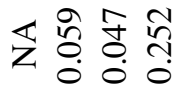

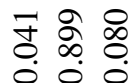

त̦

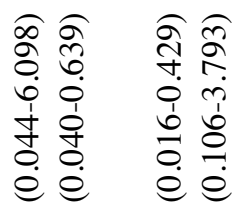

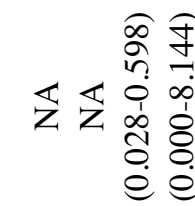

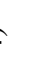

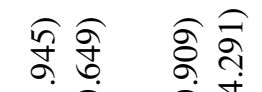

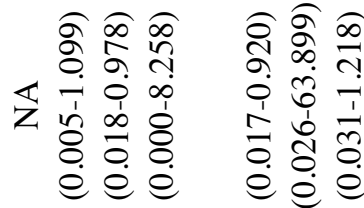

สิ ิิ

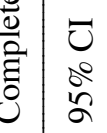

ํํㄹำ

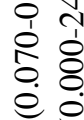

守守石

刍

$\begin{array}{lll}n & 0 & 0 \\ & 0 & 0 \\ 0 & 0 & 0 \\ 0 & 0\end{array}$

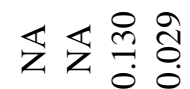

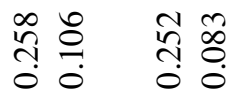

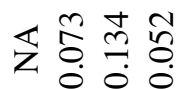

ิㅗำ

$\stackrel{\ominus}{\ominus}$

플

gin $\curvearrowleft g$

o요 in

$\stackrel{\infty}{\circ}$

लै के ๆ

mซํำสतำ 


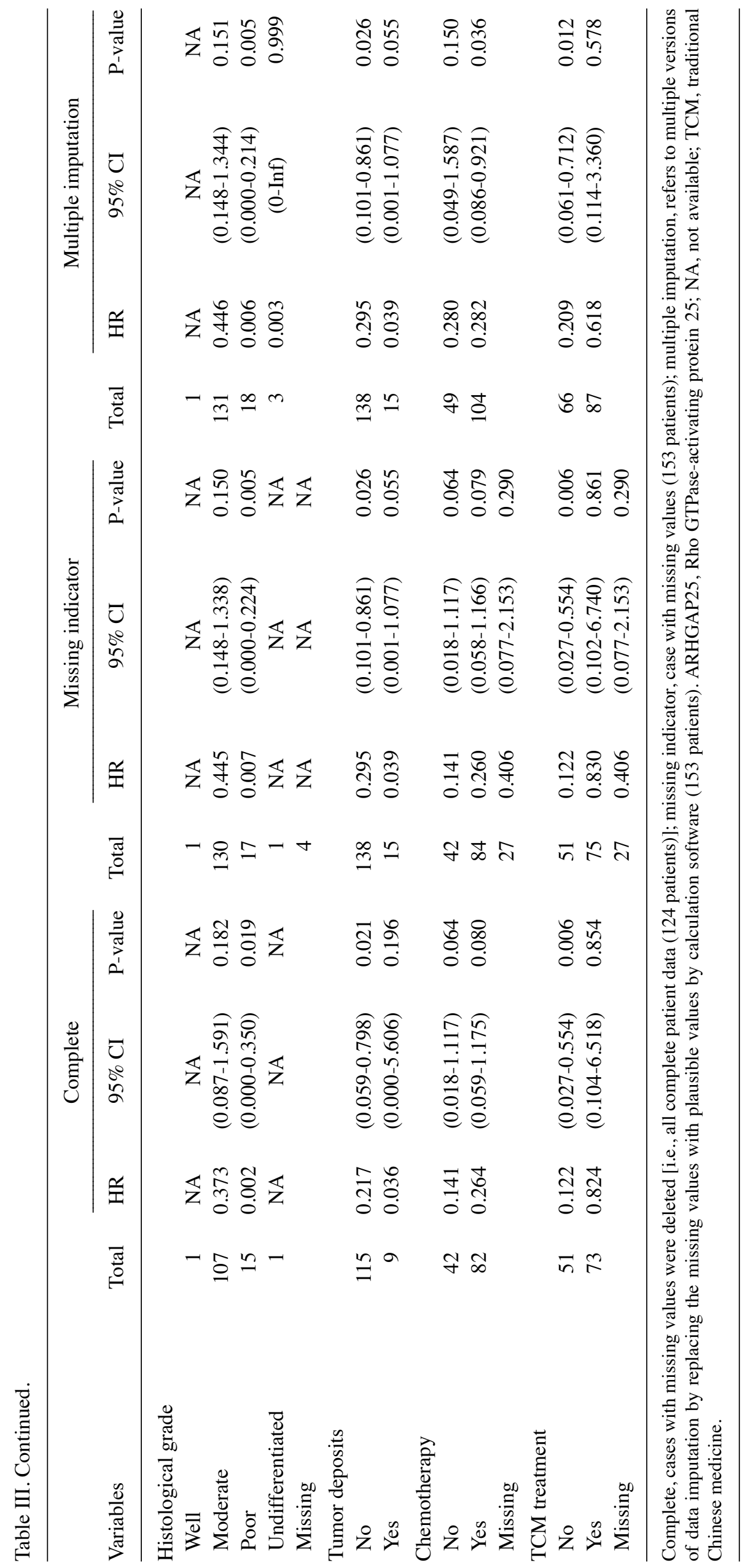


stage, lymph node metastasis, or TNM stage III. In addition, our findings indicated that ARHGAP25 expression significantly predicted OS in patients with TNM stage IV. Overall, ARHGAP25 exhibited certain advantages in extending OS.

However there are some limitations in the present study. The association between serum CEA level, R0 surgical resection, surgical type (open, laparoscopic, robotic), postoperative complications and OS were not included in this study. The detailed reasons are the following: i) Clinically, $5 \mathrm{ng} / \mathrm{ml}$ is generally regarded as the cut-off value of CEA expression. The association between CEA expression and OS was analyzed by the log-rank test of survival analysis, and the result was statistically significant, the lower the CEA expression, the longer the OS (37). In addition, considering that the influence of surgery and other therapeutic means on CEA, dynamically change this value, CEA was not included in this study. ii) In our study, 153 patients mainly underwent surgical R0 resection. Only 4 cases underwent R1 resection and 3 cases underwent $\mathrm{R} 2$ resection, thus the remaining patients underwent $\mathrm{R} 0$ resection. As is well known, the OS of patients following $\mathrm{R} 0$ resection is longer than other surgical resection methods $(38,39)$, and our study results also reached the same conclusion. Cox regression of the survival analysis was used to compare the effects of the other two surgical types (open and laparoscopic) on OS, and the results were not statistically significant. Thus, they were not included in this study. iii) The number of postoperative complications in the 153 patients was extremely low; 5 cases in total including postoperative bleeding, postoperative wound infection, vaginal fistula, wound infection, and peritoneal adhesions. Therefore, they were not included in the comparison.

In conclusion, the present study demonstrated the association between ARHGAP25 expression and prognosis. Therefore, ARHGP25 could be considered as a potential indicator for a favorable prognosis of CRC, particularly in patients with poor prognostic factors.

\section{Acknowledgements}

Not applicable.

\section{Funding}

This research was supported by the National Natural Science Foundation of China (grant nos. 81603548 and 82174450).

\section{Availability of data and materials}

The raw data supporting the findings of this article are available from the authors, without undue reservation.

\section{Authors' contributions}

YuZ and YL acquired, analyzed, and interpreted the data, drafted the article, and critically revised the study for important intellectual content. In addition, they contributed equally to this work. YiZ collected and analyzed the data. $\mathrm{XZ}$ performed the pathological and immunohistochemical analysis. LT conceived and designed the study, helped in the manuscript preparation and English language editing and critically revised it for important intellectual content. MY analyzed and interpreted the data. YuZ and LT confirm the authenticity of all the raw data. All authors read and approved the final manuscript.

\section{Ethics approval and consent to participate}

The present study was approved by the Institutional Review Board of Longhua Hospital Shanghai University of Traditional Chinese Medicine (Approval no. 2018LCSY004). The study was performed in full agreement with the national ethical and regulatory guidelines, and with the 1964 Declaration of Helsinki and its later amendments or comparable ethical standards. All patients signed a written informed consent to participate in this study.

\section{Patient consent for publication}

Not applicable.

\section{Competing interests}

The authors declare that they have no competing interests.

\section{References}

1. Bray F, Ferlay J, Soerjomataram I, Siegel RL, Torre LA, Torre LA and Jemal A: Global cancer statistics 2018: GLOBOCAN estimates of incidence and mortality worldwide for 36 cancers in 185 countries. CA Cancer J Clin 68: 394-424, 2018.

2. Katoh $\mathrm{M}$ and Katoh M: Identification and characterization of ARHGAP24 and ARHGAP25 genes in silico. Int J Mol Med 14: 333-338, 2004.

3. Lindner SE, Egelston CA, Huard SM, Lee PP and Wang LD: Arhgap25 deficiency leads to decreased numbers of peripheral blood B cells and defective germinal center reactions. Immunohorizons 4: 274-281, 2020.

4. Csépányi-Kömi R, Sirokmány G, Geiszt M and Ligeti E: ARHGAP25, a novel Rac GTPase-activating protein, regulates phagocytosis in human neutrophilic granulocytes. Blood 119: 573-582, 2012.

5. Csépányi-Kömi R, Wisniewski É, Bartos B, Lévai P, Németh T, Balázs B, Kurz AR, Bierschenk S, Sperandio M and Ligeti E: Rac GTPase activating protein ARHGAP25 regulates leukocyte transendothelial migration in mice. J Immunol 197: 2807-2815, 2016.

6. Tao L, Zhu Y, Gu Y, Zheng J and Yang J: ARHGAP25: A negative regulator of colorectal cancer (CRC) metastasis via the Wnt/ $\beta$-catenin pathway. Eur J Pharmacol 858: 172476, 2019.

7. Jensen EC: Quantitative analysis of histological staining and fluorescence using ImageJ. Anat Rec (Hoboken) 296: 378-381, 2013.

8. Varghese F, Bukhari AB, Malhotra R and De A: IHC profiler: An open source plugin for the quantitative evaluation and automated scoring of immunohistochemistry images of human tissue samples. PLoS One 9: e96801, 2014.

9. Choi J, Dekkers OM and le Cessie S: A comparison of different methods to handle missing data in the context of propensity score analysis. Eur J Epidemiol 34: 23-36, 2019.

10. Pedersen AB, Mikkelsen EM, Cronin-Fenton D, Kristensen NR, Pham TM, Pedersen L and Petersen I: Missing data and multiple imputation in clinical epidemiological research. Clin Epidemiol 9: 157-165, 2017.

11. White IR and Carlin JB: Bias and efficiency of multiple imputation compared with complete-case analysis for missing covariate values. Stat Med 29: 2920-2931, 2010.

12. Wong CK, Lam CL, Poon JT, McGhee SM, Law WL, Kwong DL, Tsang $\mathbf{J}$ and Chan P: Direct medical costs of care for Chinese patients with colorectal neoplasia: A health care service provider perspective. J Eval Clin Pract 18: 1203-1210, 2012.

13. Vega FM and Ridley AJ: Rho GTPases in cancer cell biology. FEBS Lett 582: 2093-2101, 2008. 
14. Etienne-Manneville S and Hall A: Rho GTPases in cell biology. Nature 420: 629-635, 2002.

15. Bernards A: GAPs galore! A survey of putative Ras superfamily GTPase activating proteins in man and Drosophila. Biochim Biophys Acta 1603: 47-82, 2003.

16. Tcherkezian J and Lamarche-Vane N: Current knowledge of the large RhoGAP family of proteins. Biol Cell 99: 67-86, 2007.

17. Thuault S, Comunale F, Hasna J, Fortier M, Planchon D, Elarouci N, De Reynies A, Bodin S, Blangy A and Gauthier-Rouvière C: The RhoE/ROCK/ARHGAP25 signaling pathway controls cell invasion by inhibition of Rac activity. Mol Biol Cell Cell 27: 2653-2661, 2016.

18. Xu K, Liu B and Ma Y: The tumor suppressive roles of ARHGAP25 in lung cancer cells. Onco Targets Ther 12: 6699-6710, 2019.

19. Derwinger K, Carlsson G and Gustavsson B: A study of lymph node ratio as a prognostic marker in colon cancer. Eur J Surg Oncol 34: 771-775, 2008

20. Mehrkhani F, Nasiri S, Donboli K, Meysamie A and Hedayat A: Prognostic factors in survival of colorectal cancer patients after surgery. Colorectal Dis 11: 157-161, 2009.

21. Nitsche U, Friess H, Agha A, Angele M, Eckel R, Heitland W, Jauch KW, Krenz D, Nüssler NC, Rau HG, et al: Prognosis of mucinous and signet-ring cell colorectal cancer in a population-based cohort. J Cancer Res Clin Oncol 142: 2357-2366, 2016

22. Serra-Rexach JA, Jimenez AB, Garcia-Alhambra MA, Pla R, Vidán M, Rodriguez P, Ortiz J, García-Alfonso P and Martín M: Differences in the therapeutic approach to colorectal cancer in young and elderly patients. Oncologist 17: 1277-1285, 2012.

23. Majek O, Gondos A, Jansen L, Emrich K, Holleczek B Katalinic A, Nennecke A, Eberle A and Brenner H; GEKID Cancer Survival Working Group: Sex differences in colorectal cancer survival: Population-based analysis of 164,996 colorectal cancer patients in Germany. PLoS One 8: e68077, 2013.

24. Compton C, Fenoglio-Preiser CM, Pettigrew N and Fielding LP: American joint committee on cancer prognostic factors consensus conference: Colorectal working group. Cancer 88: 1739-1757, 2000.

25. Newland RC, Dent OF, Lyttle MN, Chapuis PH and Bokey EL: Pathologic determinants of survival associated with colorectal cancer with lymph node metastases. A multivariate analysis of 579 patients. Cancer 73: 2076-2082, 1994.

26. Shepherd NA, Baxter KJ and Love SB: The prognostic importance of peritoneal involvement in colonic cancer: A prospective evaluation. Gastroenterology 112: 1096-1102, 1997.

27. Park YJ, Park KJ, Park JG, Lee KU, Choe KJ and Kim JP Prognostic factors in 2230 Korean colorectal cancer patients: Analysis of consecutively operated cases. World J Surg 23: 721-726, 1999.

28. Vasile L, Olaru A, Munteanu M, Pleşea IE, Surlin V and Tudoraşcu C: Prognosis of colorectal cancer: Clinical, pathological and therapeutic correlation. Rom J Morphol Embryol 53: 383-391, 2012

29. Missiaglia E, Jacobs B, D'Ario G, Di Narzo AF, Soneson C Budinska E, Popovici V, Vecchione L, Gerster S, Yan P, et al: Distal and proximal colon cancers differ in terms of molecular, pathological, and clinical features. Ann Oncol 25: 1995-2001, 2014.
30. Tejpar S, Stintzing S, Ciardiello F, Tabernero J, Van Cutsem E, Beier F, Esser R, Lenz HJ and Heinemann V: Prognostic and predictive relevance of primary tumor location in patients With RAS wild-type metastatic colorectal cancer: Retrospective analyses of the CRYSTAL and FIRE-3 trials. JAMA Oncol 3 : 194-201, 2017.

31. Derwinger K, Kodeda K, Bexe-Lindskog E and Taflin H: Tumour differentiation grade is associated with TNM staging and the risk of node metastasis in colorectal cancer. Acta Oncol 49: 57-62, 2010.

32. Kanazawa T, Watanabe T, Kazama S, Tada T, Koketsu S and Nagawa H: Poorly differentiated adenocarcinoma and mucinous carcinoma of the colon and rectum show higher rates of loss of heterozygosity and loss of E-cadherin expression due to methylation of promoter region. Int J Cancer 102: 225-229, 2002.

33. Mirkin KA, Kulaylat AS, Hollenbeak CS and Messaris E: Prognostic significance of tumor deposits in stage III colon cancer. Ann Surg Oncol 25: 3179-3184, 2018.

34. Nagayoshi K, Ueki T, Nishioka Y, Manabe T, Mizuuchi Y, Hirahashi M, Oda Y and Tanaka M: Tumor deposit is a poor prognostic indicator for patients who have stage II and III colorectal cancer with fewer than 4 lymph node metastases but not for those with 4 or more. Dis Colon Rectum 57: 467-474, 2014.

35. Liu X, Xiu LJ, Jiao JP, Zhao J, Zhao Y, Lu Y, Shi J, Li YJ, Ye M, Gu YF, et al: Traditional Chinese medicine integrated with chemotherapy for stage IV non-surgical gastric cancer: A retrospective clinical analysis. J Integr Med 15: 469-475, 2017.

36. Yang X, Hao J, Zhu CH, Niu YY, Ding XL, Liu C and Wu XZ: Survival benefits of western and traditional Chinese medicine treatment for patients with pancreatic cancer. Medicine (Baltimore) 94: e1008, 2015.

37. Tsouma A, Aggeli C, Lembessis P, Zografos GN, Korkolis DP Pectasides D, Skondra M, Pissimissis N, Tzonou A and Koutsilieris M: Multiplex RT-PCR-based detections of CEA, CK20 and EGFR in colorectal cancer patients. World J Gastroenterol 16: 5965-5974, 2010.

38. Diaconescu A, Alexandrescu S, Ionel Z, Zlate C, Grigorie R, Brasoveanu V, Hrehoret D, Ciurea S, Botea F, Tomescu D, et al: Resection of concomitant hepatic and extrahepatic metastases from colorectal cancer-a worthwhile operation? Chirurgia (Bucur) 112: 673-682, 2017.

39. Luo M, Chen SL, Chen J, Yan H, Qiu Z, Chen G, Lu L and Zhang F: Resection vs ablation for lesions characterized as resectable-ablative within the colorectal liver oligometastases criteria: A propensity score matching from retrospective study. PeerJ 8: e8398, 2020.

This work is licensed under a Creative Commons Attribution-NonCommercial-NoDerivatives 4.0 International (CC BY-NC-ND 4.0) License. 